\title{
Forehead reconstruction using modified double- opposing rotation-advancement flaps for severe skin necrosis after filler injection
}

\author{
Jinwoo Kim, \\ Woosuk Hwang \\ Department of Plastic and Reconstructive \\ Surgery, Busan Paik Hospital, Inje University \\ School of Medicine, Busan, Korea
}

\begin{abstract}
Varying degrees of complications can occur after hyaluronic acid filler injections. Tissue necrosis due to interruption of the vascular supply is an early complication that can be severe. If the site of tissue necrosis due to the filler injection is the forehead, successfully reconstructing the region without distorting the key landmarks is challenging. We describe the case of a 50-year-old man who experienced widespread forehead skin necrosis after hyaluronic acid filler injection in the glabellar area. We successfully covered the forehead area with a $3 \times 4-\mathrm{cm}^{2}$ midline necrotic tissue using the modified double-opposing rotationadvancement flap method. Although modified double-opposing rotation-advancement flap closure has the disadvantage of leaving a longer scar compared to conventional doubleopposing rotation-advancement flap closure, the additional incision line made along the superior border of the eyebrow aids in camouflaging the scar and decreases eyebrow distortion. Therefore, it is believed that the modified double-opposing rotation-advancement flap technique is an excellent tool for providing adequate soft tissue coverage and minimal free margin distortion when reconstructing widespread skin necrosis in the central mid-lower forehead that can occur after filler injection in the glabellar area.
\end{abstract}

Keywords: Forehead / Surgical flaps / Dermal filler

\section{INTRODUCTION}

Soft tissue augmentation using hyaluronic acid (HA) fillers is widely used to decrease facial lines and improve the appearance of soft tissues. However, varying degrees of complications can occur after HA filler injection [1,2]. Tissue necrosis due to interruption of the vascular supply is an early complication that can be severe [3].

In the present study, we describe a patient with widespread skin necrosis after HA filler injection in the glabellar area. Additionally, we describe a unique flap design inspired by the double-opposing rotation-advancement flap method used by Ransom and

Correspondence: Jinwoo Kim

Department of Plastic and Reconstructive Surgery, Busan Paik Hospital, Inje University School of Medicine, 75 Bokji-ro, Busanjin-gu, Busan 47392, Korea

E-mail: jinooda@hanmail.net

Received November 9, 2017 / Revised March 8, 2018 / Accepted March 8, 2018
Jacono [4]. Using modified double-opposing rotation-advancement flaps, it is possible to achieve adequate soft tissue coverage and minimal free margin distortion when reconstructing widespread skin necrosis in the central mid-lower forehead that can occur after filler injection in the glabellar area.

\section{CASE REPORT}

A 50-year-old man presented as an outpatient to the Plastic Surgery Department of Busan Paik Hospital. His main symptom was worsening of skin necrosis immediately after a filler injection that was administered 3 weeks prior at a local clinic to treat frown lines in the glabellar area. Tissue necrosis progressed despite the initial treatment including massage, warm compression, and hyaluronidase injection (Fig. 1). The patient was treated as an outpatient for 
2 weeks to confirm the necrosis margin and to facilitate wound healing. Wound healing progressed, but secondary healing produced unacceptable scars. Therefore, he was admitted to the hospital for reconstructive surgery (Fig. 2).

The patient underwent surgery under systemic anesthesia on day 1 of admission. To effectively cover the $3 \times 4-\mathrm{cm}^{2}$ midline fore-

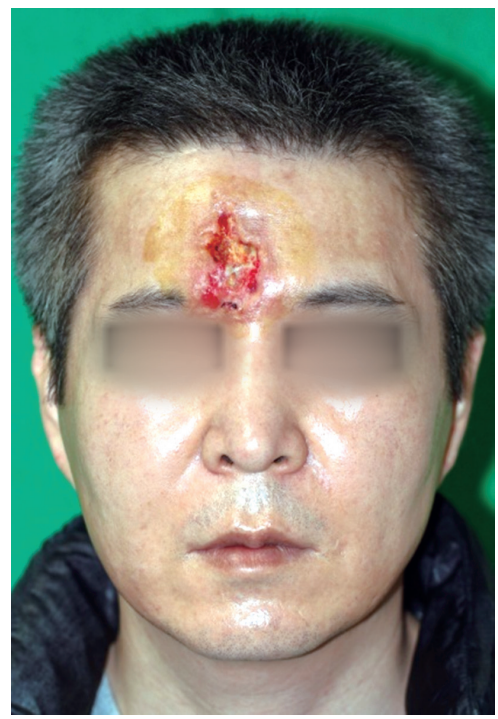

Fig. 1. Approximately $3 \times 4-\mathrm{cm}^{2}$ skin defect due to necrosis on day 1 . Skin necrosis occurred after the patient was administered a filler injection in the glabellar area 3 weeks previously.

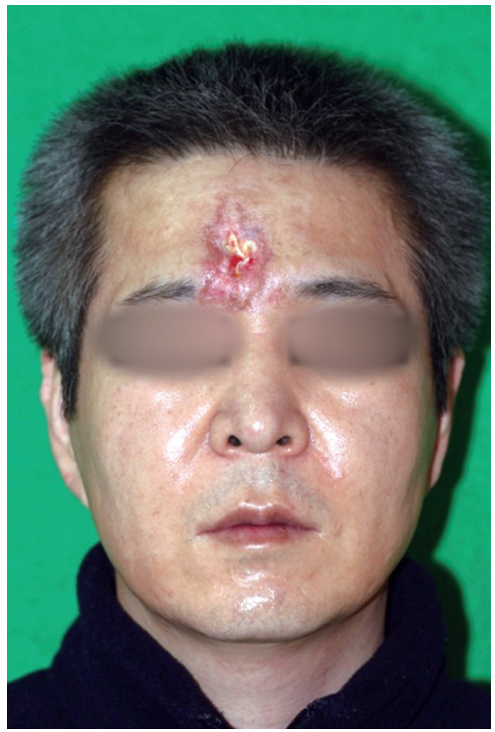

Fig. 2. The necrosis margin was defined and the wound had partially healed at 2 weeks after the first visit. It was considered an unacceptable scar. head skin defect that was created after removing necrotic tissue and poorly healed granulation tissues, modified double-opposing rotation-advancement flaps were designed. After adequate debridement, the flaps were raised on the subgaleal plane. The upper area of the eyebrow was released while partially releasing the skin of the lower area. The elevated lower left flap was rotated to cover the defect, and the upper left and upper right flaps were advanced to the defect (Fig. 3).
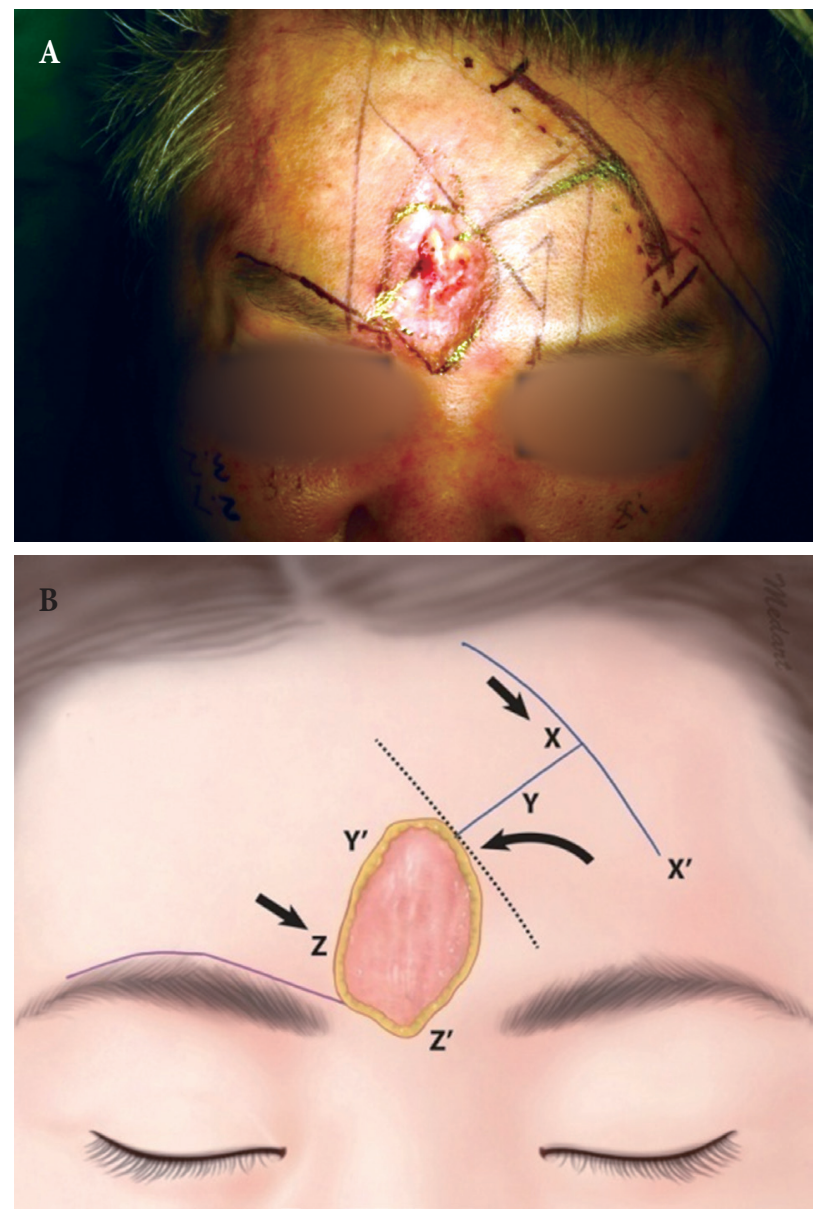

Fig. 3. Modified double-opposing rotation-advancement flaps. (A) During surgery. (B) The conventional double-opposing rotationadvancement flap design (blue lines) was created to prevent eyebrow distortion while filling large defects by making an additional incision (purple line) along the supra eyebrow line. During the procedure, one flap is rotated toward the primary defect ( $\mathrm{Y}$ to $\left.\mathrm{Y}^{\prime}\right)$, and the contralateral flaps are advanced to the secondary defect (X to X', Z to Z'). A dotted line is a tangent on the curvilinear defect margin drawn parallel to the schematic pathway of frontal branch of the facial nerve. Blue lines make a sort of capital " $\mathrm{T}$ " with slightly curved crossbar (one line is drawn at approximately $60^{\circ}-75^{\circ}$ from the dotted line, the other line is drawn roughly parallel to the dotted line, with a gentle curve). 
Suturing using absorbable polyfilament (Vicryl; Ethicon, Somerville, NJ, USA) with some pieces of fascia was performed in deeper skin layers. A second suture layer was placed on the epidermis using a nonabsorbable monofilament (Mersilk, Ethicon). Penrose drains were inserted (Fig. 4). There were no complications. The patient was discharged 10 days after surgery. It was confirmed that forehead reconstruction was successful at 3 months postoperatively (Fig. 5).

\section{DISCUSSION}

Vascular compromise after soft tissue augmentation with injectable facial fillers is a complication that should be addressed postoperatively. In particular, facial areas such as the glabellar area have
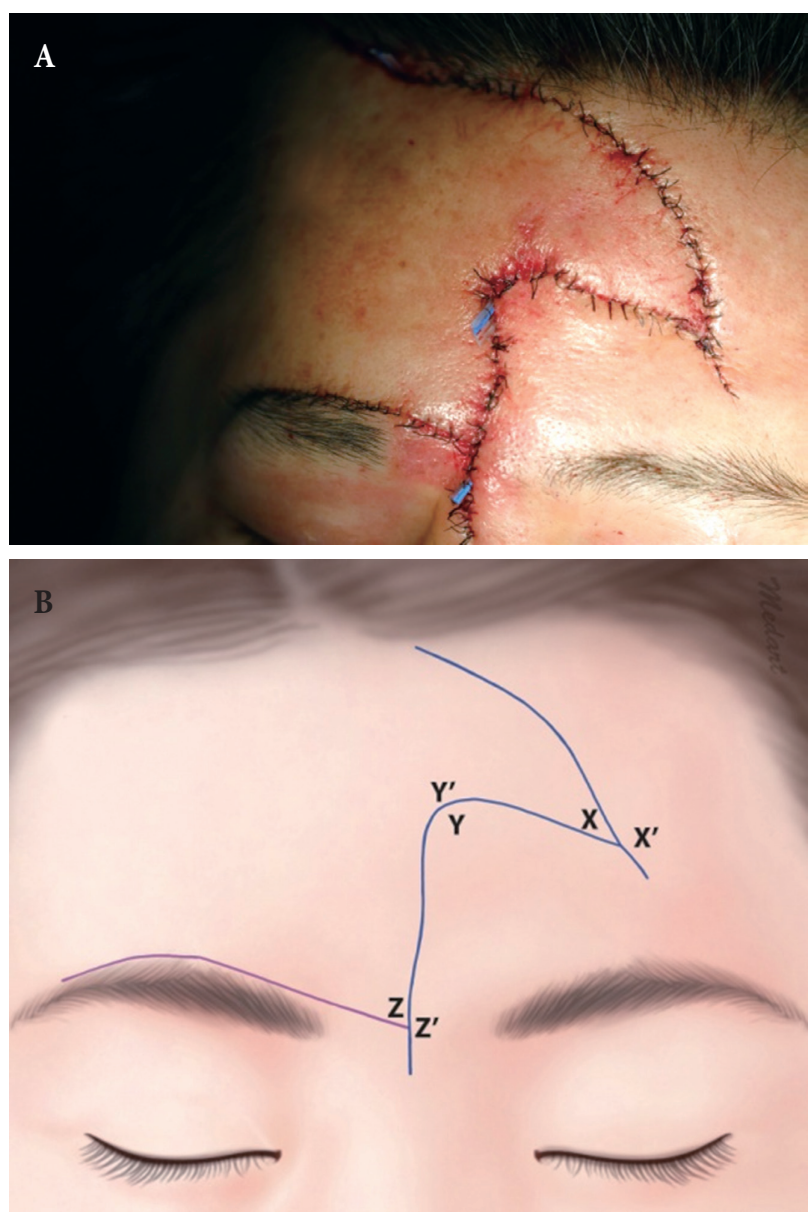

Fig. 4. Final closure. (A) During surgery. (B) The elevated lower left flap was rotated to cover the defect (Y-Y'), and the upper left and upper right flaps were advanced to the defect (X-X', Z-Z').



Fig. 5. Forehead reconstruction was successful at 3 months after surgery. Minimal eyebrow distortion occurred after surgery. The scar at the superior border of the eyebrow was almost invisible.

been reported to be at higher risk for necrosis due to compromised blood flow attributable to the vascular anatomy of the area [5].

It has been reported that when filler-induced soft tissue necrosis is clinically suspected, various treatment approaches such as heparin, corticosteroid, hyaluronidase, platelet-rich plasma, and others are as effective as early interventions [5-7]. When necrosis has progressed past the acute phase, reconstructive surgery should be attempted.

The forehead is a facial region with distinct boundaries defined by the hairline superiorly, eyebrows and frontonasal groove inferiorly, and temporal ridges laterally. Therefore, reconstructing the region successfully without distorting the key landmarks is challenging [8]. Additionally, serious consideration should be given to midline forehead reconstruction to prevent eyebrow distortion (elevation of the medial eyebrows and medial displacement of the eyebrows) and to preserve motor and sensory nerve functions [9].

Regarding reconstruction of skin defects of the lower forehead or mid forehead near the glabellar area, Quatrano et al. [10] proposed that primary repair should be performed if the reconstruction area is smaller than $2 \mathrm{~cm}^{2}$, the modified Burow's advancement flap or A-to-T flap should be used if the area is approximately $2-5 \mathrm{~cm}^{2}$, and a bilateral advancement flap or full- 
thickness skin graft should be used if the area is larger than $5 \mathrm{~cm}^{2}$. However, when full-thickness necrosis of the central mid-lower forehead skin occurs in an area as large as $10 \mathrm{~cm}^{2}$ (as in our patient), a bilateral advancement flap can distort the eyebrows, and a skin graft may lead to wound contraction, poor color, and mismatched texture.

Ransom and Jacono [4] presented the double-opposing rotation-advancement flap method modified from the orticochea flap method as a versatile reconstructive option for small, medium, and large forehead defects. However, there were limitations regarding eyebrow distortion and covering forehead defects larger than $10 \mathrm{~cm}^{2}$.

Accordingly, inspired by the double-opposing rotation-advancement flaps, we performed an additional incision along the superior border of the eyebrow contralateral to that used during the conventional approach. This unique flap design led to successful reconstruction because durable soft tissue covering a forehead skin defect as large as $10 \mathrm{~cm}^{2}$ was created during a single stage without altering the eyebrow position.

Although modified double-opposing rotation-advancement flaps have the disadvantage of leaving a longer scar compared to conventional approaches, the additional incision line made along the superior border of the eyebrow was camouflaged by the eyebrow. Therefore, it is believed that modified double-opposing rotation-advancement flaps are an excellent tool for central midlower forehead skin defect reconstruction.

\section{CONFLICT OF INTEREST}

No potential conflict of interest relevant to this article was reported.

\section{PATIENT CONSENT}

The patients provided written informed consent for the publication and the use of their images.

\section{REFERENCES}

1. Andre P. Hyaluronic acid and its use as a "rejuvenation" agent in cosmetic dermatology. Semin Cutan Med Surg 2004;23:218-22.

2. Bachmann F, Erdmann R, Hartmann V, Wiest L, Rzany B. The spectrum of adverse reactions after treatment with injectable fillers in the glabellar region: results from the Injectable Filler Safety Study. Dermatol Surg 2009;35 Suppl 2:1629-34

3. Schanz S, Schippert W, Ulmer A, Rassner G, Fierlbeck G. Arterial embolization caused by injection of hyaluronic acid (Restylane). Br J Dermatol 2002;146:928-9.

4. Ransom ER, Jacono AA. Double-opposing rotation-advancement flaps for closure of forehead defects. Arch Facial Plast Surg 2012;14:342-5.

5. Hirsch RJ, Cohen JL, Carruthers JD. Successful management of an unusual presentation of impending necrosis following a hyaluronic acid injection embolus and a proposed algorithm for management with hyaluronidase. Dermatol Surg 2007;33:357-60.

6. Tracy L, Ridgway J, Nelson JS, Lowe N, Wong B. Calcium hydroxylapatite associated soft tissue necrosis: a case report and treatment guideline. J Plast Reconstr Aesthet Surg 2014;67:564-8.

7. Kang BK, Kang IJ, Jeong KH, Shin MK. Treatment of glabella skin necrosis following injection of hyaluronic acid filler using platelet-rich plasma. J Cosmet Laser Ther 2016;18:111-2.

8. De Abullarade J. Aesthetic considerations in forehead reconstruction in skin carcinoma. J Cancer Ther 2013;4:20-3.

9. Baker SR. Local flaps in facial reconstruction. 3rd ed. Philadelphia: Elsevier; 2014.

10. Quatrano NA, Dawli TB, Park AJ, Samie FH. Simplifying forehead reconstruction: a review of more than 200 cases. Facial Plast Surg 2016;32:309-14. 